\title{
Radioisotope Distribution Program Progress Report for May 1976
}

\section{E. Lamb}

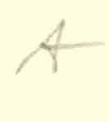

\section{OAK RIDGE NATIONAL LABORATORY}




\section{DISCLAIMER}

This report was prepared as an account of work sponsored by an agency of the United States Government. Neither the United States Government nor any agency Thereof, nor any of their employees, makes any warranty, express or implied, or assumes any legal liability or responsibility for the accuracy, completeness, or usefulness of any information, apparatus, product, or process disclosed, or represents that its use would not infringe privately owned rights. Reference herein to any specific commercial product, process, or service by trade name, trademark, manufacturer, or otherwise does not necessarily constitute or imply its endorsement, recommendation, or favoring by the United States Government or any agency thereof. The views and opinions of authors expressed herein do not necessarily state or reflect those of the United States Government or any agency thereof. 


\section{DISCLAIMER}

Portions of this document may be illegible in electronic image products. Images are produced from the best available original document. 
Printed in the United States of America. Available from

National Technıcal Iıluı ination Serviré

U.S. Department of Commerce

5285 Port Royal Road, Springfield, Virginia 22161

Price: Printed Copy $\$ 3.50$; Microfiche $\$ 2.25$

This report was prepared as an account of work sponsored by the United States Government. Neither the United States nor the Energy Research and Development Administration/United States Nuclear Regulatory Commission, nor any of their employees, nor any of their contractors, subcontractors, or their employees, makes any warranty, express or implied, or assumes any legal liability or responsibility for the accuracy, completeriess or ucefulness of any information, apparatus, product or process disclosed, or represents that its use would not infringe privately owned riylıts. 
Contract No. W-7405-eng-26

OPERATIONS DIVISION

\section{RADIOISOTOPE DISTRIBUTION PROGRAM \\ PROGRESS REPORT FOR MAY 1976}

JULY 1976

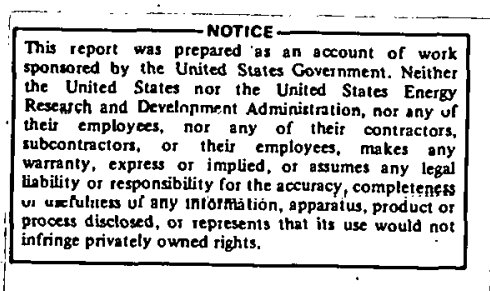

NOTICE This document contains information of a preliminary nature and was prepared primarify for internal use at the Osk Ridge National Laboratory. It is subject to revision or correction and thorofore does not represent a final report.

OAK RIDGE NATIONAL LABORATORY

Oak Ridge, Tennessee 37830

operated by

UNION CARBIDE CORPORATION

for the

ENERGY RESEARCH AND DEVELOPMENT ADMINISTRATION 
THIS PAGE

\section{WAS INTENTIONALLY \\ LEFT BLANK}


CONTENTS

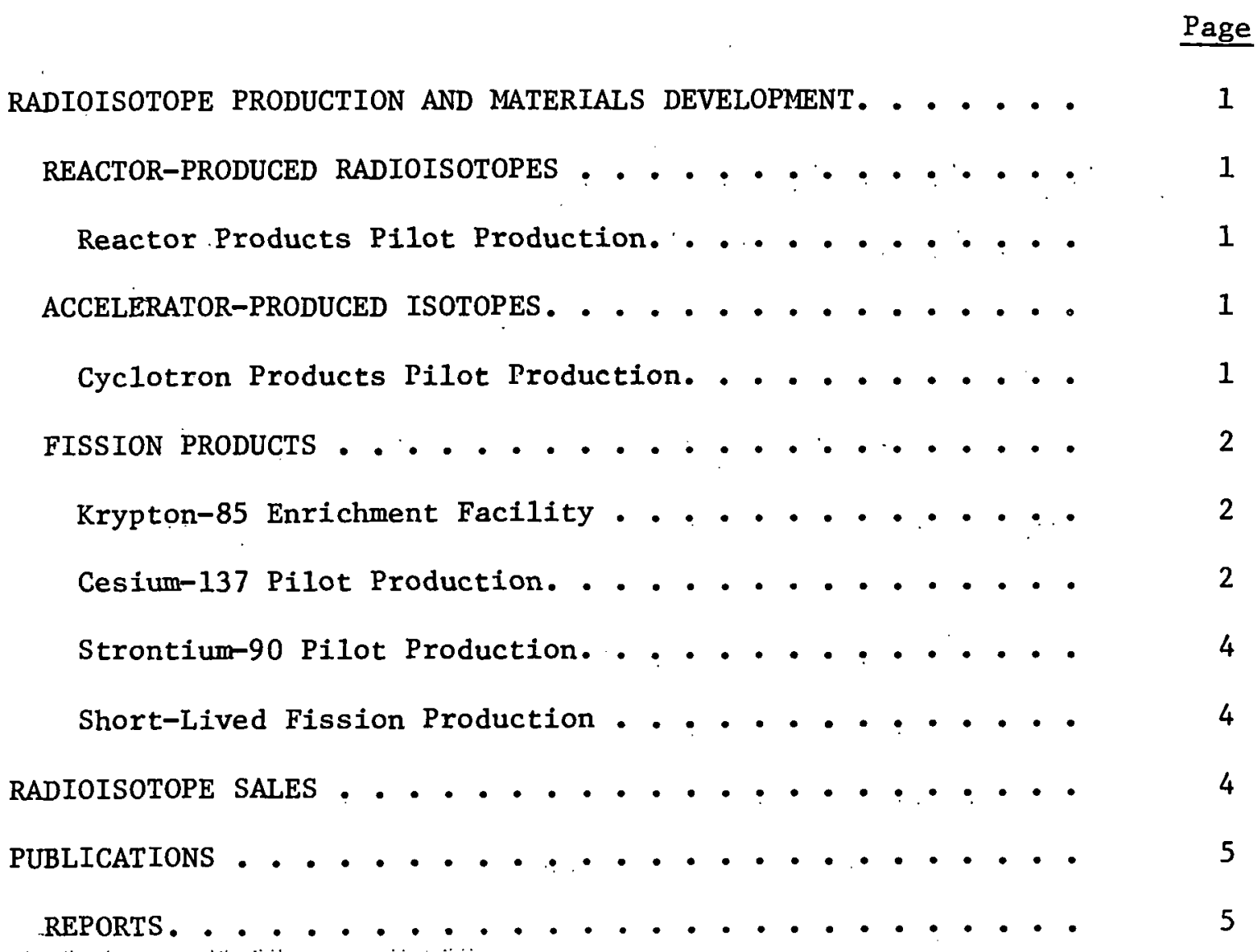


RADIOISOTOPE DISTRIBUTION PROGRAM

PROGRESS REPORT FOR MAY 1976

$$
\text { E. Lamb }
$$

RADIOISOTOPE PRODUCTION AND. MATERIALS DEVELOPMENT

\section{REACTOR-PRODUCED RADIOISOTOPES}

Reactor Products Pilot. Production (R. W. Schaich)

(Production and Inventory Accounts.)

\begin{tabular}{lc}
$\frac{\text { Processed Units }}{\text { Radioisotope }}$ & Amount (mCi) \\
\hline Calcium-47 & 16 \\
Copper-67 & 9
\end{tabular}

\section{ACCELERATOR-PRODUCED ISOTOPES}

Cyclotron Products Pilot Production (M. R. Skidmore)

(Production and Inventory Accounts)

May 1976 ORNL 86-Inch Cyclotron runs for ORNL and non-ORNL programs are given in Table 1.

Table 1. Cyclotron Irradiations and Runs for May 1976

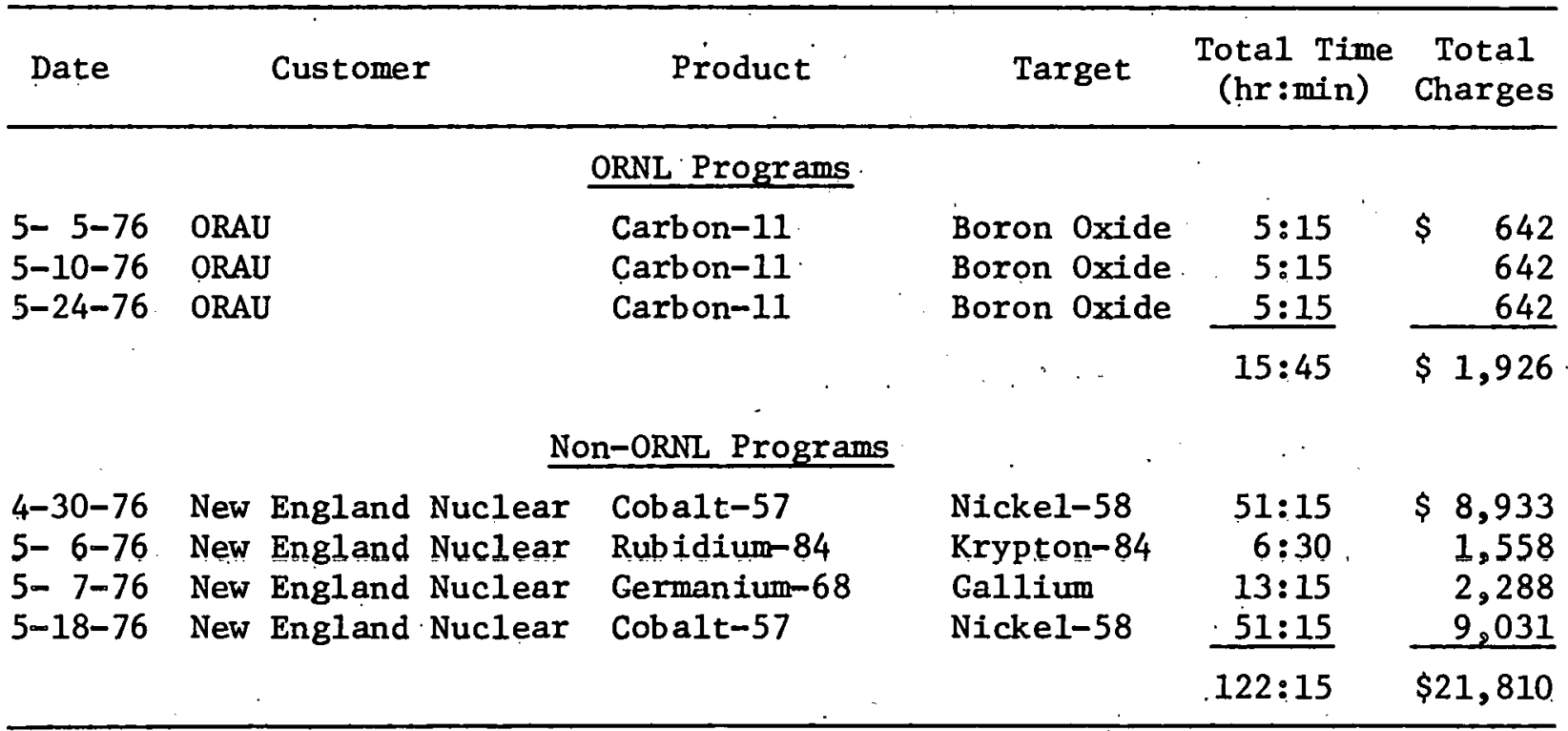




\section{Cyclotron Operations}

The mercury rectifiers in the cubicles (the d.c. high voltage power supplies for oscillator) were replaced with six stacks of solid state devices in 1968. On May 14, 1976, master cubicle 96 failed and all six stacks of rectifiers in the cubicle were found to have failed. The rectifiers were replaced and the rest of the cubicle was checked. We had smooth operations for the rest of the month.

\section{FISSION PRODUCTS}

Krypton-85 Enrichment Facility (R. W. Schaich)

The ${ }^{85} \mathrm{Kr}$ enrichment colums operated satisfactorily during the month of May. Unloading operations will be initiated after the installation of a new unloading station. This station is being designed and should be in operation by July 1976 .

Cesium-137 Pilot Production (R. W. Schaich) (Production and Inventory Accounts)

\section{Process Status}

The analytical data on the purified powder batches recovered from the WESF container was completed and the product powder averaged $24.4 \mathrm{Ci}$ per gram. A total of $42,164 \mathrm{Ci}$ was recovered from the WESF container, which represents a yield of $91.7 \%$.

\section{Operational Summary}

\section{Product Inventory}

(Decay calculated through April 30, 1975)

Inventory Material

Amount (Ci)

In-Process

Cesium-137 chloride powder

Special form cans and Fabricated Sources

Total Inventory Material $42 ; 500$

Non-Inventory Materlal

$\Lambda$ mounc (C1)

$\begin{array}{lr}\text { Material returned or stored for customer } & \\ \text { Puerto Rico sources } & 8,200 \\ \text { Lockheed } & 20,300 \\ \text { AECL powder } & 73,800 \\ \text { Radiation Resources } & 34,600 \\ \text { Minn. Mining \& Mfg. Company } & 9,500 \\ \text { Gamma Industries } & 8,600 \\ \text { J. L. Shepherd } & 13,400 \\ \text { Total Non-Inventory Material } & 168,400 \\ \text { TOTAL INVENTORY AND NON-INVENTORY MATERIAL } & 210,900\end{array}$


Fabrication Summary

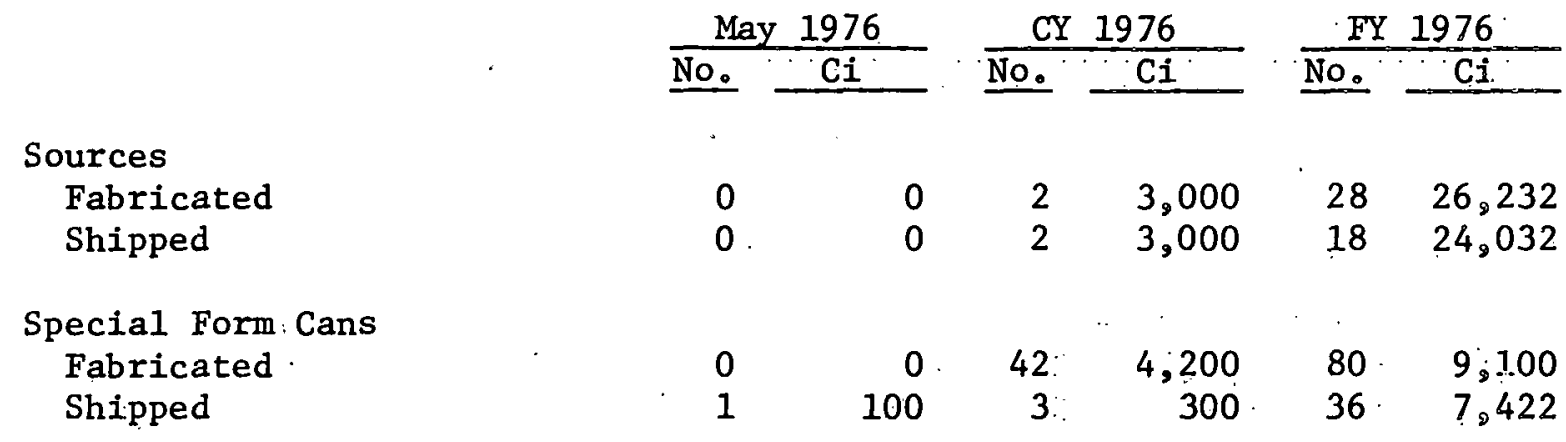

3. Current Orders

All orders on hand have been completed and the material placed into storage awaiting receipt of release for the material.

Strontium-90 Pilot Production (R. W. Schaich) (Production and Inventory Accounts)

1. Process Status

The ${ }^{90} \mathrm{Sr}$ process and manipulator cells are being decontaminated under the ERDA Decommissioning Program. The ${ }^{90} \mathrm{Sr}$ powder was removed from the FPDL, encapsulated, and stored for future orders.

Product Inventory

(Decay calculated through April 30, 1975)

Inventory Material

Amount (Ci).

${ }^{90} \mathrm{Sr}$ titanate powder $( \pm 5 \%)$

476,000

Sources in fabrication

RCA source

${ }^{90} \mathrm{Sr}$ silicate powder

57,800

28,200

Stock powder cans

4,000

Total Inventory Material

566,000

Non-Inventory Material

Amount (Ci)

FPDL recovery materlal

18,200

Quehanna recovery material

44,400

Weather Bureau source

11,800

SNAP-7B

161,500

$S N A P-7 C$

25,400

SNAP - 7:D

147,800

SNAP material purchase.

$\underline{256,600}$

- Total Non-Inventory Material 665,700

TOTAL INVENTORY AND NON-INVEETORY MATERIAL

$1,231,700$

${ }^{a}$ Strontium-90 purchased under DRRD program. 
Fabrication Sưmary

\begin{tabular}{|c|c|c|c|c|c|c|}
\hline & \multicolumn{2}{|c|}{ May 1976} & \multicolumn{2}{|c|}{ CY 1976} & \multicolumn{2}{|c|}{ FY 1976} \\
\hline & No. & $C i$ & No. & $\mathrm{Ci}$ & No.: & $\mathrm{Ci}$ \\
\hline \multicolumn{7}{|l|}{ Sources } \\
\hline Fabriçated & 0 & 0 & 0 & 0 & 0 & 0 \\
\hline Shipped & 0 & 0 & 0 & 0 & 0 . & 0 \\
\hline \multicolumn{7}{|c|}{ Special Form Cans } \\
\hline Fabricated & 0 & 0 & 0 & 0 & 0 & 0 \\
\hline Shipped & 1. & 100 & 5 & 234 & 9 & 604 \\
\hline
\end{tabular}

Short-Lived Fission Production (R. W. Schaich)

(Production and Inventory Accounts).

\begin{tabular}{lcr} 
Isotope & Number of Batches & Amount \\
\cline { 2 - 3 } Iodine-131 & 1 & 50 \\
Xenon-133 & 2 & 1300
\end{tabular}

\section{RADIOISOTOPE SALES}

J. E. Rațedge

Shipments made during the month that may be of interest are listed below:

Customer

Isotope

Amount

Large Quantities

New England Nuclear Corporation

American Atomics

ICN Pharmaceuticals

Saunders-Roe, England

Self-Powered Lighting

$\begin{array}{ll}\text { Tritium } & 6,000 \mathrm{Cl} \\ \text { Tritium } & 6,000 \mathrm{Ci} \\ \text { Tritium } & 1,000 \mathrm{Ci} \\ \text { Tritium } & 5,000 \mathrm{Ci} \\ \text { Tritium } & 2,000 \mathrm{Ci}\end{array}$

\section{Withdrawn Items}

$\mathrm{K}-25$ Plant

Cleveland General Hospital
Iodine-131

Iodine-131
$5 \mathrm{mCi}$

$50 \mathrm{mCi}$

\section{Items Used in Cooperative Programs}

University of Southern California

P1atinum-195m

$18 \mathrm{mCi}$

The radioisotope sales and shipments for the first eleven months of FY 1975 and FY 1976 are given in Table 2. 
Tab le 2. Radioisotope: Sales and. Shipments

\begin{tabular}{|c|c|c|c|c|}
\hline Item & \multicolumn{2}{|c|}{$\begin{array}{l}7-1-74 \text { thru } \\
5-31-75\end{array}$} & \multicolumn{2}{|c|}{$\begin{array}{c}7-1-75 \text { thru } \\
5-31-76\end{array}$} \\
\hline $\begin{array}{l}\text { Inventory items } \\
\text { Major products } \\
\text { Radioisotope services } \\
\text { Cyclotron irradiations } \\
\text { Miscellaneous processed materials } \\
\text { Packing and Shipping }\end{array}$ & $\$$ & $\begin{array}{r}342,635 \\
92,390 \\
81,199 \\
90,689 \\
130,701 \\
69,015 \\
\end{array}$ & $\$$ & $\begin{array}{r}272,335 \\
49,516 \\
80,319 \\
164,801 \\
51,593 \\
102,110 \\
\end{array}$ \\
\hline Total & $\$$ & 806,629 & $\$$ & 720,674 \\
\hline Number of shipments & & 1,457 & & 1,567 \\
\hline
\end{tabular}

\section{PUBLICATIONS}

\section{REPORTS}

E. Lamb, Radioisotope Distribution Progrom Progress Report for Apriz 1976, ORNL/TM-5537, Oak Ridge National Laboratory (June 1976). 
THIS PAGE

\section{WAS INTENTIONALLY LEFT BLANK}


INTERNAL DISTRIBUTION

\author{
1. E. E. Beauchamp \\ 2. T. A. Butler \\ 3. F. N. Case. \\ 4. W. R. Casto \\ 5. J. A. Cox \\ 6. R. F. Hibbs \\ 7. E. Lamb \\ 8. H. H. Nichol \\ 9. C. L. Ottinger \\ 10. J. K. Poggenburg \\ 11. H. Postma
}
12. M. E. Ramsey
13. J. E. Ratledge
14. C. R. Richmond
15. A. F. Rupp
16. R. W. Schaich
17. M. R. Skidmore
18. M. J. Skinner
19-20. Central Research Library
21-22. Laboratory Records Department
23. Laboratory Records - RC

\section{EXTERNAL DISTRIBUTION}

25. B. J. Dropesky, LASL, Los Alamos, New Mexico

26-27. J. H. Jarrett, PNL, Richland, Washington

28. D. K. Jones, Richland Operations Office, Richland, Washington

29. L. M. Knights, Atlantic Richfield Hanford Company, Richland, Washington

30. J. N. Maddox, ERDA-DBER, Washington, D. C.

31. W. E. Mott, ERDA-DBER, Washington, D. C:

32. H. A. O'Brien, LASL, Los Alamos, New Mexico

33. F. J. Skozen (Krizek), Argonne Cancer Research Hospital, Chicago

34. L. G. Stang; Jr., BNL, New York

35-36. R. W. Wood, ERDA-DBER, Washington, D. C.

37. Donner Laboratory Library, University of California, Berkeley, Calif., 94720

38. Research and Technical Support Division, ORO

39-40. Technical Information Center 